\title{
ORDER STABILITY ANALYSIS OF RECIPROCAL JUDGMENT MATRIX
}

\author{
Mingzhe Wang ${ }^{\mathrm{a}}$ Danyi Wang \\ ${ }^{a}$ Huazhong University of Science and Technology, Wuhan, Hubei 430074 - P. R. China \\ mzwangpublic.wh.hb.cn \\ ${ }^{\text {b }}$ George Mason University, Fairfax, VA 22030 USA, dwang2@ gmu.edu
}

\begin{abstract}
Summary:
The analytic hierarchy process (AHP) introduced by T.L. Saaty is a well-known and popular analytic model of multi-criteria decision making. The final object of AHP is the priority ordering of the decision alternatives. However, because of a lot of uncertain factors and perturbations, the $n$ column vectors in the matrix are absolutely not consistent. So, people have to look for the column vectors in the matrix is a " close" structure to compose a close consistent matrix. The "close" here means the "order clustering" that all column vectors of a judgment matrix have a same (or near same) rank ordering sequence.

Let us suppose that each column vectors expressed in the judgment matrix as one time of judgment by decision maker and also one element in positive vector space. It is clear that a decision making process heavily depends on the rank ordering of the judgment for alternatives, associated with inconsistency, the inverse ranking inconsistency is a troublesome problem. Although a lot of works have done effectively, from the view of rank order structure of judgment matrixes, we do not catch the fancy of the favorable rank ordering for the judgment matrix completely.
\end{abstract}

For examples, the consistency levels of matrixes A is accepted, but its estimated results in table1 shows that the rank orderings estimated by different methods are not same.

Table 1: Different priority vectors and rank order of matrix A

\begin{tabular}{c|ccc|cccc}
\hline $\mathbf{A}$ & $\mathrm{A}_{1}$ & $\mathrm{~A}_{2}$ & $\mathrm{~A}_{3}$ & $\mathrm{EM}$ & NRA & LSM & LLSM \\
\hline $\mathrm{A}_{1}$ & 1 & $1 / 4$ & $1 / 3$ & $0.1261(\mathbf{1})$ & $0.1253(\mathbf{1})$ & $0.1258(\mathbf{1})$ & $0.1291(\mathbf{1})$ \\
$\mathrm{A}_{2}$ & 4 & 1 & $4 / 5$ & $0.4254(\mathbf{2})$ & $0.4591(\mathbf{3})$ & $0.4025(\mathbf{2})$ & $0.4355(\mathbf{2 . 5})$ \\
$\mathrm{A}_{3}$ & 3 & $5 / 4$ & 1 & $0.4485(\mathbf{3})$ & $0.4156(\mathbf{2})$ & $0.4717(\mathbf{3})$ & $0.4355(\mathbf{2 . 5})$ \\
\hline \multicolumn{7}{c}{$\lambda_{\max }=3.0291, \mathrm{C} \mathrm{R}=0.028<5 \%$} \\
\hline
\end{tabular}

An important basic aspect of AHP is the estimation of the priority rank ordering of the alternatives from the judgment matrix. In order to represent the decision maker's ordinal perception faithfully, let us consider the strict preference order relations such as $\mathrm{A}_{1} \succ \mathrm{A}_{2} \succ \ldots \mathrm{A}_{\mathrm{i}} \succ \ldots \succ \mathrm{A}_{\mathrm{n}}$ and the same (or indifferent ) preference order such as $\mathrm{A}_{\mathrm{k}}{ }^{\sim} \mathrm{A}_{\mathrm{k}+1}, \mathrm{k}$ ? $(1,2, \ldots, \mathrm{n}-1)$ for the set of alternatives $\left\{\mathrm{A}_{1}, \mathrm{~A}_{2}\right.$, $\left.\ldots \mathrm{A}_{\mathrm{i}}, \ldots, \mathrm{A}_{\mathrm{n}}\right\}$. One column vector of a judgment matrix $\mathrm{A}=\left(a_{i j}\right)$ is regarded as one time of judgment with the different unitage alternative $A_{i}, i ?(1,2, \ldots, n)$.

Definition 1. Any column vectors of matrix $A=\left(a_{i j}\right)$, such as column vectors $a_{i}, a_{j} \in R_{n}^{+}$, have same priority rank order if for each k, $1 ?(1,2, \ldots, \mathrm{n}), a_{k i}>a_{l i}$ and $a_{k j}>a_{l j}$ or $a_{k i} \approx a_{l i}$ and $a_{k j} \approx a_{l j}$, that means they have equivalence order relation $\mathrm{EO}$, denoted by $a_{i} \mathrm{EO} a_{j}$.

For example: $a_{2} \mathrm{EO} a_{3}$ in table 1 . 
Theorem 1. EO is a binary relation of $R_{n}^{+}$. It is also a rank equivalence relation that determines unique rank equivalent class of $R_{n}^{+}$.

Definition 2. A rank equivalent class with $\mathrm{k}$ rank positions in same order position is called a (n-k-1) dim rank equivalent class. The dimension of a strict rank equivalent class in $R_{n}^{+}$is $\mathrm{n}$.

Theorem 2. The vector space $R_{n}^{+}$holds $\mathrm{n}$ ! strict rank equivalent classes. Each one of strict rank equivalent classes adjoins (n-1) side rank equivalent classes in which only one couple rank positions holds same rank order, (n-2) side rank equivalent classes with only three same rank positions, (n-k) side rank equivalent classes with $(\mathrm{k}+1)$ same rank positions, and so on.

Definition 3. Based on one rank equivalent class, its neighbor rank equivalent class is obtained by exchanging only one couple of connecting order positions. Its side rank equivalent class is obtained by extending the same order positions without changing its other rank orderings, and all of its side rank equivalent classes construct its bound rank equivalent class.

Theorem 3. A certain strict rank equivalent class represented alternative ordering as $A_{1} \succ A_{2} \succ \ldots A_{k}$ $\succ \ldots \succ \mathrm{A}_{\mathrm{n}}$ is a $\mathrm{n}$-dim rank equivalent class, which has $2^{n-1}$ of side equivalent classes to be composed its bound equivalent class in $R_{n}^{+}$.

Definition 4. A quasi-close rank equivalent class is a set of this rank equivalent class with its bound equivalent class together.

Theorem 4. Any rank equivalent class in the vector space $R_{n}^{+}$is a convex cone.

Corollary 1. All independent column vectors of a judgment matrix compose a convex cone in $R_{n}^{+}$.

Definition 5. The order structure of a judgment matrix is said to be stable if any no-negative linear combinations of all column vectors of this judgment matrix keeps same rank order for decision alternatives.

Theorem 5. A sufficient condition for stable order structure of judgment matrix is that all of its column vectors belong to one rank equivalent class in the vector space $R_{n}^{+}$

Corollary 2. (1) The order structure of a positive reciprocal judgment matrix is strict stable if all of its column vectors belong to one strict rank equivalent class of $R_{n}^{+}$

(2) The order structure of a positive reciprocal judgment matrix is critical stable if all of its column vectors are in a side rank equivalent class of $R_{n}^{+}$

(3) The order structure of a positive reciprocal judgment matrix is stable if all of its column vectors are in a quasi-close rank equivalent class of $R_{n}^{+}$

(4) The order structure of a positive reciprocal judgment matrix is asymptotically stable if its dimension of the matrix is adequate and all of its column vectors are distributed in the region of a rank equivalent class with its adjoining classes.

Human error and various uncertain perturbations may exist in each facet of analysis process. Analytic Hierarchy Process provides a very helpful cognitive control mode to implement and preserve quality of solutions of multi-criteria decision making, but complexity of real cognitive situation is a truly very large problem. We have not solved it. Hopefully, we have set forth a good start for solution in this field. 